\title{
Correction to: Microwave Oscillators and Detectors Based on Magnetic Tunnel Junctions
}

P. K. Muduli, Raghav Sharma, Dhananjay Tiwari, Naveen Sisodia, Afshin Houshang, O. G. Heinonen, and Johan Åkerman

\section{Correction to:}

Chapter "Microwave Oscillators and Detectors Based on Magnetic Tunnel Junctions" in: W. S. Lew et al. (eds.), Emerging Non-volatile Memory Technologies, https://doi.org/10.1007/978-981-15-6912-8_1

The original version of the book was published without incorporating the chapter "Microwave Oscillators and Detectors Based on Magnetic Tunnel Junctions" corrections provided by the corresponding author. The correction chapter and book have been updated with the changes. 